Series A

\author{
I. MATHEMATICA
}

315

\title{
ZUR THEORIE DER \\ QUANTENMECHANISCHEN \\ VERTAUSCHUNGSRELATIONEN
}

\author{
von \\ YRJÖ KILPI
}


Am 9. März 1962 vorgelegt von R. und F. Nevandinna. 


\section{Einleitung}

Seitdem E. SchröDINGER im Jahre 1926 gezeigt hat, dass seine Wellenmechanik mit der Heisenbergschen Matrizenmechanik mathematisch äquivalent ist, ist es in der Quantenmechanik ein sehr interessantes Problem gewesen, alle möglichen Paare von selbstadjungierten Transformationen $P, Q$ im Hilbertschen Raum $\mathfrak{S}$ zu bestimmen, welche die Eigenschaft.

$$
P Q-Q P=-i I
$$

haben. Speziell hat man die Bedingungen untersucht, die das Transformationspaar $P, Q$ ausser (1) erfüllen muss, damit es mit dem Heisenbergschen Matrizenoperatorenpaar

(2)

$$
P_{0}=\frac{1}{i \sqrt{2}}\left|\begin{array}{rrrrr}
0 & \sqrt{1} & 0 & 0 & \ldots \\
-\sqrt{1} & 0 & \sqrt{2} & 0 & \ldots \\
0 & -\sqrt{2} & 0 & \sqrt{3} & \ldots \\
0 & 0 & -\sqrt{3} & 0 & \ldots \\
\ldots, \ldots & \ldots & \ldots & \ldots
\end{array}\right|,
$$

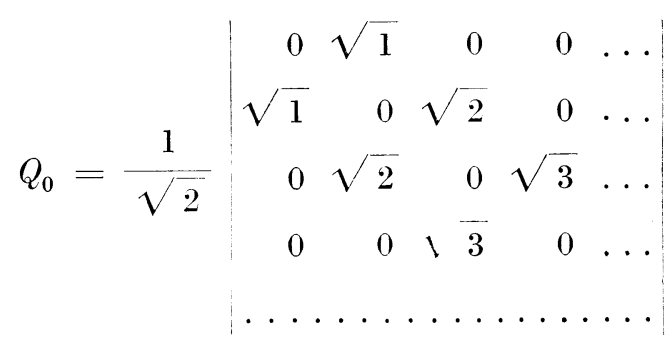
im Hilbertschen Raum der Vektoren $\varphi=\left\{\varphi_{1}, \varphi_{2}, \ldots\right\}$ mit $\sum_{\nu=1}^{\infty}\left|\varphi_{\nu}\right|^{2}<\infty$
unitär äquivalent $\left.{ }^{1}\right)$ sei $\left.^{2}\right)$.

1) Man sagt, dass zwei selbstadjungierte Transformationen $P$ und $P^{\prime}$ unitär äquivalent sind, wenn es eine solche unitäre Transformation $U$ und ihre Adjungierte $U^{*}\left(=U^{-1}\right)$ gibt, bei der $U^{*} P U=P^{\prime}$ gilt.

2) Es sei bemerkt, dass man mit den Matrizenoperatoren (2) immer ein orthonormales System $\psi_{0}, \psi_{1}, \psi_{2}, \ldots, \psi_{v}, \ldots$ in dem obenerwähnten Hilbertschen Raum folgendermassen assoziieren kann: 
v. Neumann zeigte im Jahre 1931, dass jedes der Bedingung (1) genügende Transformationspaar auch die Vertauchungsrelation von WEYL

$$
e^{i t P} e^{i s Q}=e^{i t s} e^{i s Q} e^{i t P} \quad(-\infty<s, t<\infty)
$$

erfüllt, wobei $t, s$ reelle Parameter bedeuten und $e^{i t P}$ und $e^{i s Q}$ lineare Transformationen sind, die man aus $P$ und $Q$ durch Potenzenentwicklungen formal erhalten hat (vgl. hier z.B. Foras-GeHÉr-Sz.-NAGY [2], S. 79-80), und zwar so, dass die Transformationen $P, Q$ bis auf unitäre Aquivalenz eindeutig bestimmt sind.

Die ursprüngliche Vertauschungsrelation (1) ist erst 1946 von RELLICH [4] und später 1958 von Drxmier [1] untersucht worden. Thre Resultate lassen sich folgendermassen formulieren:

Satz 1. (Dixmier [1], S. 267-268). Es seien $P_{1} \ldots, P_{n}, Q_{1}, \ldots, Q_{n}$ abgeschlossene symmetrische Transformationen im Hilbertschen Raum is, und es sei $\mathfrak{X}$ ein in $\mathfrak{H}$ überall dichter invarianter $C$ nterraum von $P_{1}, \ldots$, $P_{n}, Q_{1}, \ldots, Q_{n}$, der zu $D\left(P_{1}\right) \cap \ldots \cap D\left(P_{n}\right) \cap D\left(Q_{1}\right) \cap \ldots \cap D\left(Q_{n}\right)$ gehört, und zwar so, dass

$1^{\circ}$

$$
\begin{aligned}
& \left(P_{j} Q_{k}-Q_{k} P_{j}\right) f=-i \delta_{j k} f, \\
& \left(P_{j} P_{k}-P_{k} P_{j}\right) f=0 \\
& \left(Q_{j} Q_{k}-Q_{k} Q_{j}\right) f=0
\end{aligned}
$$

bei jedem $f$ von $\mathfrak{X}$ gilt,

$2^{\circ}$ die kleinsten abgeschlossenen linearen Fortsetzungen der auf $\mathfrak{x}$ liegenden Einschränkungen von $P_{j}^{2}+P_{k}^{2}, Q_{j}^{2}+Q_{h}^{2}, P_{j}^{2}+Q_{k}^{2}$

$(j, k=1,2, \ldots, n)$ selbstadjungiert sind.

Dann existiert eine solche Menge von gegeneinander orthogonalen abgeschlossenen linearen Unterräumen $\mathfrak{S}_{1}, \mathfrak{S}_{2}, \ldots$ deren Summe $\mathfrak{5}$ ist, mit folgenden Eigenschaften:

Man bilde die Transformationen $P^{\prime}$ und $Q^{\prime}$, deren $D$ efinitionsbereiche gemeinsam sind und zu denen alle Elemente der Reihe $\psi_{0} . w_{1}, y_{2} \ldots$. und alle thre endlichen Linearkombinationen $\sum \alpha_{v} \psi_{v}$, gehören, wo also ron den Konstanten $\alpha_{v}$, nur endlich viele von Null verschieden sind. Dann gilt

$$
\begin{aligned}
& P^{\prime} \psi_{v}=\frac{1}{i \sqrt{2}}\left(\sqrt{v} \psi_{v-1}-\sqrt{v-1} \psi_{v-1}\right), \\
& Q^{\prime} \psi_{v}=\frac{1}{\sqrt{2}}\left(\sqrt{v} \psi_{v-1}-\sqrt{v+1} \psi_{v+1}\right),
\end{aligned}
$$

wobei $\psi_{-1}=0, v=1,2, \ldots$. Dann stimmen, wie bekannt, die kleinsten linearen Fortsetzungen von $P^{\prime}$ und $Q^{\prime}$ mit den Heisenbergschen Matrizenoperatoren $P_{0}$ und $Q_{0}$ überein. 
$\mathrm{a}^{\circ} P_{1}, \ldots, P_{n}, Q_{1}, \ldots, Q_{n}$ werden von jedem $\mathfrak{\mathfrak { S }}_{\mu}(\mu=1,2, \ldots)$ reduziert.

$\mathrm{b}^{\circ}$ Das System $P_{1}, \ldots, P_{n}, Q_{1}, \ldots, Q_{n}$ ist in jedem Unterraum $\mathfrak{S}_{\mu}$ mit dem Heisenbergschen Operatorensystem im Falle von $n$ Freiheitsgraden unitär äquivalent.

Weiterhin sind $P_{1}, \ldots, P_{n}, Q_{1}, \ldots, Q_{n}$ und die kleinsten linearen abgeschlossenen Fortsetzungen ihrer auf $\mathfrak{X}$ liegenden Einschränkungen selbstadjungiert.

Bei RELLICH entspricht der Bedingung $2^{\circ}$ die Annahme, dass die Transformation $A=\sum\left(P_{j}^{2}+Q_{j}^{2}\right)$ zerlegbar ist, d.h. eine Spektralschar $E_{\lambda}$ mit den gleichzeitigen Eigenschaften existiert:

(a) für jedes $g$ und für jedes endliche $\lambda, \lambda^{\prime}$ gehört $\left(E_{i^{\prime}}-E_{\lambda}\right) g$ zu $D(A)$

(b) für jedes $g$ aus $D(A)$ gilt $A f=\int_{-\infty}^{+\infty} \lambda d E_{i} f$.

In dem Falle $n=1$ zeigte DixmזER, dass aus dieser Rellichschen Annahme die Bedingung $2^{\circ}$ folgt und dass darum sein Ergebnis das von RELLICH enthält. Dixmier aber erwähnte, er könne in dem Falle $n>1$ keinen Beweis dafür geben, dass aus der obigen Rellichschen Vermutung die Bedingung $2^{\circ}$ folgen kann.

Es erhebt sich die Frage, warum das Dixmiersche Ergebnis das quantenmechanische Vertauschungsproblem so erschöpfend in dem Falle von einem Freiheitsgrade, aber nicht so vollständig in dem von mehreren Freiheitsgraden löst. Die Antwort auf diese Frage ist offenbar darin zu suchen, dass Dixmier in $2^{\circ}$ zuviel angenommen hat.

Es ist daher der Zweck dieser Arbeit, zu zeigen, dass man, ohne die Behauptungen des Satzes 1 zu beschränken, statt der Vermutung $2^{\circ}$ nur anzunehmen braucht, dass

$2^{\circ}$ die kleinsten abgeschlossenen linearen Fortsetzungen der auf $\mathfrak{X}$ liegenden Einschränkungen von $P_{j}^{2}+Q_{j}^{2}(j=1,2, \ldots, n)$ selbstadjungiert sind.

Wenn man dies gezeigt hat und also in Satz 1 die Vermutung $2^{\circ}$ durch $2^{\circ \circ}$ ersetzt, so enthält ein solcher Satz das Rellichsche Ergebnis auch in dem Falle mehrerer Freiheitsgrade, wie man aus der Untersuchung von Dixmier [1] schliessen kann.

Um das Obige zu beweisen, betrachten wir zuerst normale und hypermaximale normale Transformationen im Hilbertschen Raum, die folgendermassen definiert sind:

Eine in $\mathfrak{H}$ dicht definierte lineare Transformation $N$ ist normal, wenn $D(N) \subseteq D\left(N^{*}\right)$ gilt und die Einschränkung $\bar{N}$ von $N^{*}$ auf $D(N)$ mit $N$ metrisch gleich ist, d.h., dass $|N f|=\bar{N} f \mid$ für jedes $f$ von $D(N)$ gilt. Falls speziell $D(N)=D\left(N^{*}\right)$ ist, heisst $N$ hypermaximal normal. 
Wir werden in $\S 1$ Satz 2 zeigen, dass alle Transformationen

$$
P_{\mu} \pm i P_{v}, P_{\mu} \pm i Q_{\lambda}, Q_{\mu} \pm i Q_{\nu}(\mu, v, \lambda=1,2, \ldots, n ; \mu \neq \lambda)
$$

normal sind und dass die kleinsten linearen abgeschlossenen Fortsetzungen ihrer auf $\mathfrak{X}$ liegenden Einschränkungen hypermaximal normal sind. Die Beweisidee liegt darin, dass jeder der Transformationen (4) eine eindeutige Lösung des komplexen Momentenproblems (vgl. hier KILPI [3]) entspricht und dass dieser Lösungsfunktion wiederum eine eindeutig bestimmte hypermaximale normale Transformation entspricht. Nach diesen Betrachtungen ist dann ohne Schwierigkeiten zu schliessen, dass man, wie wir es in $\S 2$ getan haben, die Dixmiersche Bedingung $2^{\circ}$ durch die von uns gemachte Annahme $2^{\circ \circ}$ ersetzen kann.

Es sei erwähnt, dass im J. 1960 FoIAs, GeHER und Sz. -NAGY [2] auf sehr elegante Weise den Zusammenhang zwischen den Vertauschungsrelationen (1) und (3) in dem Falle eines Freiheitsgrades untersucht haben. Den komplizierteren Fall $n>1$ haben sie aber öffentlich nie behandelt.

\section{§ 1. Fortsetzungsprozess}

1.1 Wir erwähnen hier zunächst einige Hilfssätze, die wir im folgenden brauchen werden.

Hilfssatz 1. (Vgl. Stone [5], Satz VI, S. 10-13). Es sei $f(x)$ eine Funktion, die durch die Bedingungen

$$
\begin{aligned}
& f(x)=0, \text { wenn }-\infty<x<a \text { und } b<x<\infty, \\
& f(a)=f(b)=\frac{1}{2}, \\
& f(x)=1, \text { wenn } a<x<b,
\end{aligned}
$$

definiert ist. Dann konvergiert die Reihe

$$
c_{0} e^{-x^{2}} H_{0}(x)+c_{1} e^{-x^{2}} H_{1}(x)+\ldots+c_{n} e^{-x^{2}} H_{n}(x)+\ldots,
$$

wobei $H_{n}(x)(n=0,1,2, \ldots)$ die Polynome von Hermite bedeuten und wobei

$$
c_{n}=\frac{1}{2^{n} n ! \sqrt{\pi}} \int_{a}^{b} e^{-x^{2}} H_{e}(x) d x
$$

gilt, bei jedem Wert von $x$ gegen die Funktion $e^{-x^{2}} f(x)$, und sogar so, dass die Konvergenz in den Intervallen $-\infty<x<a-\varepsilon, a+\varepsilon<x<b-\varepsilon$, $b+\varepsilon<x<\infty$, wobei $\varepsilon$ eine beliebig kleine positive Konstante bedeutet, gleichmässig ist. Die Reihe 


$$
c_{0} H_{0}(x)+c_{1} H_{1}(x)+\ldots+c_{n} H_{n}(x)+\ldots
$$

konvergiert dann überall gegen $f(x)$, und die Konvergenz ist gleichmässig in jedem endlichen Intervall, innerhalb dessen a und $b$ nicht liegen. ${ }^{1}$ )

Wenn wir nun $a=0$ wählen, können wir durch die Substitution $x=(z-\alpha)(\bar{z}-\bar{\alpha})$ den Hilfssatz 1 auch für Funktionen vom komplexen Parameter $z$ folgendermassen verallgemeinern.

Hilfssatz 2. Es sei $f(z)$ eine Funktion von $z$ und seiner Konjugierten $\bar{z}$, die durch die Bedingungen

$$
\begin{aligned}
f(z) & =0, \text { wenn } & & |z-\alpha|>b, \\
f(z) & =\frac{1}{2}, \text { wenn } & & |z-\alpha|=b, \\
f(z) & =1, \text { wenn } & & |z-\alpha|<b,
\end{aligned}
$$

definiert ist. Dann konvergieren die Reihen

$$
\begin{gathered}
c_{0} H_{0}((z-\alpha)(\bar{z}-\bar{\alpha}))+c_{1} H_{1}((z-\alpha)(\bar{z}-\bar{\alpha}))+\ldots \\
\ldots+c_{n} H_{n}((z-\alpha)(\bar{z}-\bar{\alpha}))+\ldots
\end{gathered}
$$

gegen die Funktion $f(z)$ bei jedem Wert von $z$, und zwar so, dass die Konvergenz in jedem beschränkten einfach zusammenhängenden Teil der komplexen Ebene, in dessen inneren Punkten die Bedingung $|z-\alpha|=b$ nicht gilt, gleichmässig ist.

Weiterhin konvergieren die Reihen

$$
\begin{gathered}
c_{0} e^{-(z \bar{z})^{2}} H_{0}((z-\alpha)(\bar{z}-\bar{\alpha}))+c_{1} e^{(-z \bar{z})^{2}} H_{1}((z-\alpha)(\bar{z}-\bar{\alpha}))+\ldots \\
\ldots+c_{n} e^{-(z \bar{z})^{2}} H_{n}((z-\alpha)(\bar{z}-\overline{\bar{\alpha}}))+\ldots
\end{gathered}
$$

für jeden Wert $z$ gegen die Funktion $e^{-(z \bar{z})^{2}} f(z)$, und zwar so, dass die Konvergenz in den Gebieten $\infty>|z-\alpha| \geqq b+\varepsilon$ und $b-\varepsilon \geqq|z-\alpha|$, wobei $\varepsilon$ eine beliebig kleine positive Zahl bedeutet, gleichmässig ist.

1.2. Wir sind jetzt in der Lage, den folgenden Satz zu beweisen:

Satz 2. Es sei $\mathfrak{H}$ ein Hilbertscher Raum, und es seien $P$ und $Q$ zwei selbstadjungierte Transformationen mit den Definitionsbereichen $D(P)$ und $D(Q)$ in $\mathfrak{S}$. Es sei weiterhin $\mathfrak{X}$ ein in $\mathfrak{S}$ überall dichter Unterraum, der zu der Menge $D(P) \cap D(Q)$ gehört, und zwar so, dass $\mathfrak{X}$ ein invarianter Unterraum von $P$ und $Q$ ist.

1) Es sei bemerkt, dass Stone den Hilfssatz 1 nur in dem Falle $b=\infty$ bewiesen hat. Den obigen Hilfssatz erhält man jedoch unmittelbar, wenn man die Funktionen $f_{1}(x)$ und $f_{\mathbf{2}}(x)$, die durch die Bedingungen $f_{1}(x)=0$ bzw. $f_{\mathbf{2}}(x)=0$, wenn $-\infty$ $<x<a$ bzw. $-\infty<x<b, \quad f_{1}(a)=f_{\mathbf{2}}(b)=\frac{1}{2}$, und $f_{1}(x)=1$ bzw. $f_{\mathbf{2}}(x)=1$, wenn $a<x<\infty$ bzw. $b<x<\infty$, definiert sind, und die gemäss (1.2) ihnen entsprechenden Reihenentwicklungen subtrahiert. 
Man bezeichne mit $P^{\prime}$ bzw. $Q^{\prime}$ die Einschränkung von $P$ bzw. $Q$ auf $\mathfrak{X}$. Falls die kleinsten abgeschlossenen linearen Fortsetzungen von $P^{\prime}$ und $Q^{\prime}$ selbstadjungiert sind und falls $P^{\prime}$ und $Q^{\prime}$ in dem Sinne miteinander vertauschbar sind, dass die Transformationen $P^{\prime}+i Q^{\prime}$ und $P^{\prime}-i Q^{\prime}$ normal sind, so sind die kleinsten abgeschlossenen linearen Fortsetzungen von $P^{\prime}+Q^{\prime}$ und $P^{\prime}-i Q^{\prime}$ hypermaximale normale Transformationen.

Beweis. Weil $\mathfrak{X}$ ein invarianter Unterraum der Operatoren $P^{\prime}$ und $Q^{\prime}$ ist, gehören mit $f$ auch die Elemente $P f=P^{\prime} f$ und $Q f=Q^{\prime} f$ zu $\mathfrak{X}$. Durch Induktion schliessen wir dann, dass auch $P^{\prime m} Q^{\prime n} f(m, n=0,1,2, \ldots)$ existiert und zu $\mathfrak{X}$ gehört. Da $P^{\prime}+i Q^{\prime}$ normal ist, können wir auch setzen

$$
P^{m} Q^{\prime n} f=Q^{\prime n} P^{\prime m} f
$$

Weiterhin genügt $Q^{\prime n} f$ der Bedingung

$$
Q^{\prime n} f=\int_{-\infty}^{+\infty} y^{n} d E_{y} f
$$

wobei $E_{y}$ die Spektralschar von $Q$ bedeutet, und das Element $P^{\prime m} Q^{\prime n} f$ der Bedingung

$$
P^{\prime: n} Q^{\prime n} f=\int_{+\infty}^{+\infty} x^{m} d E_{x} \int_{+\infty}^{+\infty} y^{m} d E_{y} f=\int_{-\infty}^{\infty} \int_{-\infty}^{\infty} x^{m} y^{n} d E_{x} E_{y} f
$$

wobei $E_{x}$ die Spektralschar von $P$ ist und wobei das letzte Doppelintegral bedeutet, dass man zuerst mit $y$ und dann mit $x$ integrieren muss. Da $P^{\prime}+i Q^{\prime}$ normal ist, so gilt auch

$$
P^{\prime m} Q^{\prime n} f=\int_{-\infty}^{+\infty} \int_{-\infty}^{+\infty} x^{m} y^{n} d E_{x} E_{y} f=\int_{-\infty}^{+\infty} \int_{-\infty}^{+\infty} x^{m} y^{n} d E_{y} E_{x} f=Q^{\prime n} P^{\prime m} f .
$$

Wenn wir nun das Element

$$
\left(P^{\prime}+i Q^{\prime}\right)^{m}\left(P^{\prime}-i Q^{\prime}\right)^{n} f=\left(P^{\prime}-i Q^{\prime}\right)^{n}\left(P^{\prime}+i Q^{\prime}\right)^{m} f
$$

betrachten, so können wir entsprechend

$$
\begin{aligned}
& \left(P^{\prime}+i Q\right)^{m}\left(P^{\prime}-i Q^{\prime}\right)^{n} f=\int_{-\infty}^{+\infty} \int_{-\infty}^{-\infty} z^{m} \bar{z}^{n} d E_{x} E_{y} f= \\
& =\int_{-\infty}^{+\infty} \int_{-\infty}^{+\infty} z^{m} \bar{z}^{n} d E_{y} E_{x} f=\left(P^{\prime}-i Q^{\prime}\right)^{n}\left(P^{\prime}+i Q^{\prime}\right)^{m} f
\end{aligned}
$$

setzen, wobei $z=x+i y$ und $z=x-i y$ bezeichnet worden ist.

Allgemein erhalten wir 


$$
\begin{gathered}
R\left(P^{\prime}\right) f=\int_{-\infty}^{+\infty} R(x) d E_{x} f, \quad S\left(Q^{\prime}\right) f=\int_{-\infty}^{+\infty} S(y) d E_{y} f \\
T\left(P^{\prime}+i Q^{\prime}, P^{\prime}-i Q^{\prime}\right) f=\int_{-\infty}^{+\infty} \int_{-\infty}^{+\infty} T(z, \bar{z}) d E_{x} E_{y} f= \\
=\int_{-\infty}^{+\infty} \int_{-\infty}^{+\infty} T(z, \bar{z}) d E_{x} E_{y} f
\end{gathered}
$$

wobei $R(x), S(y)$ und $T(z, \bar{z})$ beliebige Polynome der entsprechenden Argumente $x, y, z$ und $\bar{z}$ und $R\left(P^{\prime}\right), S\left(Q^{\prime}\right)$ und $T\left(P^{\prime}+i Q^{\prime}, P^{\prime}-i Q^{\prime}\right)$ Polynome der entsprechenden Transformationen $P^{\prime}, Q^{\prime}, P^{\prime}+i Q$ und $P^{\prime}-i Q^{\prime}$ bedeuten.

Bezeichnen wir als $K$ ein beliebiges beschränktes abgeschlossenes einfach zusammenhängendes Gebiet der komplexen Ebene, dessen Rand keinen gemeinsamen Punkt mit den Punktspektra von $P^{\prime}+i Q^{\prime}$ und $P^{\prime}-i Q^{\prime}$ hat. Es sei weiterhin $L_{1}, L_{2}, \ldots, L_{m}$ eine solche Menge von punktfremden Kreisen mit den Mittelpunkten $a_{1}, a_{2}, \ldots, a_{m}$ und den Radien $\varrho_{1}, \varrho_{2}, \ldots, \varrho_{m}$, die sämtlich zu $K$ gehören, so dass ihre Ränder keine gemeinsamen Punkte mit den Punktspektra von $P^{\prime}+i Q^{\prime}$ und $P^{\prime}-i Q^{\prime}$ haben. Die Folge $L_{1}, L_{2}, \ldots, L_{m}$ sei weiterhin so gewählt, dass die Entfernung eines beliebigen Punktes $z_{0}$ von $K$ bis zu dem nächsten Punkt $z$ mit den Eigenschaften $\left|z-a_{\mu}\right| \leqq \varrho_{\mu}$, wobei $\mu$ eine der Zahlen $1,2, \ldots, m$ ist, kleiner als eine beliebig kleine positive Konstante $\varepsilon^{\prime}$ ist, sobald $m$ grösser ist als eine gewisse positive Zahl $m\left(\varepsilon^{\prime}\right)$.

Nach Hilfssatz 2 können wir dann ein solches Polynom $P_{\mu}(z, \bar{z})$ von $z$ und $\bar{z}$ finden, bei dem die Funktion $e^{-(\bar{z})^{2}} P_{u}(z, \bar{z})$ die Bedingungen

$$
\begin{aligned}
& \left|e^{-(z \bar{z})^{2}} P_{\mu}(z, \bar{z})-1\right|<\frac{\varepsilon}{2^{\mu+2}} \text {, wenn }\left|z-a_{\mu}\right| \leqq \varrho_{u}-\delta_{\mu} \text {, } \\
& e^{-(z \bar{z})^{2}} P_{\mu}(z, \bar{z})-\frac{\varrho_{u}+\delta_{\mu}-z-a_{u} !}{2 \delta_{\mu}}<\frac{\varepsilon}{2^{\mu+2}} \text {, wenn } \\
& \varrho_{\mu}-\delta_{\mu} \leqq\left|z-a_{\mu}\right| \leqq \varrho_{\mu}+\delta_{\mu}, \\
& \left|e^{-(z \bar{z})^{2}} P_{\mu}(z, \bar{z})\right|<\frac{\varepsilon}{2^{\mu+2}} \text {, wenn } \varrho_{\mu}+\delta_{\mu} \leqq\left|z-a_{\mu}\right| \text {. }
\end{aligned}
$$

erfüllt; hierbei bedeutet $\varepsilon$ eine beliebig kleine positive Konstante, $\varrho_{\mu}$ eine positive und $a_{\mu}$ eine komplexe Zahl und $\delta_{\mu}$ eine kleine positive Zahl, die so klein gewählt worden ist, dass das Gebiet $\varrho_{\mu}+\delta_{\mu} \geqq\left|z-a_{\mu}\right| \geqq$ 
$\varrho_{\mu}-\delta_{\mu}(\mu=1,2, \ldots)$ keinen Punkt der Punktspektra von $N=P^{\prime}+i Q^{\prime}$ und $\bar{N}=P^{\prime}-i Q$ enthält.

Bezeichnen wir jetzt mit $\varphi_{m}(z, \bar{z})$ die Funktion

$$
\varphi_{m}(z, \bar{z})=\sum_{\mu=1}^{m} P_{\mu}(z, \bar{z}) e^{--(z \bar{z})^{2}},
$$

so bemerken wir nach dem obigen, dass $\varphi_{m}(z, \bar{z})$ in den Kreisgebieten $\left|z-a_{\mu}\right|<\varrho_{\mu}-\delta_{\mu}$, unabhängig vom Werte $m$, sich von 1 weniger als $\frac{\varepsilon}{4}$ unterscheidet, und in dem Gebiet, wo die Bedingungen $\left|z-a_{\mu}\right|>$ $\varrho_{\mu}+\delta_{\mu}(\mu=1,2, \ldots, m)$ gelten, seinem absoluten Betrag nach kleiner als $\frac{\varepsilon}{4}$ ist.

Sei jetzt $f$ ein beliebiges Element von $\mathfrak{X}$. Bezeichnen wir jetzt mit $g$ das Element $g=e^{-\frac{(N \bar{N})^{2}}{2}} f$ (vgl. hier z.B. STone [6], S. 283-6). Wir haben dann

$$
\begin{aligned}
& \left(\sum_{\mu=1}^{m} P_{\mu}(N, \bar{N}) f, f\right)=\left(\sum_{\mu=1}^{m} P_{\mu}(N, \bar{N}) e^{-\frac{(z \bar{z})^{2}}{2}} f, e^{-\frac{(z \bar{z})^{2}}{2}} f\right)= \\
& =\int_{-\infty}^{+\infty} \int_{-\infty}^{+\infty} \sum_{\mu=1}^{m} P_{\mu}(z, \bar{z}) e^{-(z \bar{z})^{2}} d\left(E_{x} E_{y} f, f\right)= \\
& =\int_{-\infty}^{+\infty} \int_{-\infty}^{+\infty} \varphi_{m}(z, \bar{z}) d\left(E_{x} E_{y} f, f\right)=\int_{-\infty}^{+\infty} \int_{-\infty}^{+\infty} \varphi_{m}(z, \bar{z}) d\left(E_{y} E_{x} f, f\right),
\end{aligned}
$$

wobei die letzte Gleichung aus der Normalität von $N$ folgt (vgl. hier (1.4)).

Aus (1.5) und (1.6) folgt, dass $\varphi_{m}(z, \bar{z})$ in den Gebieten $\varrho_{\mu}+\delta_{\mu} \geqq$ $\left|z-a_{\mu}\right| \geqq \varrho_{\mu}-\delta_{\mu}(\mu=1,2, \ldots m)$ die Bedingung $\left|\varphi_{m}(z, \bar{z})\right|<1$ erfüllt. Da die Transformationen $P^{\prime}+i Q^{\prime}$ und $P^{\prime}-i Q^{\prime}$ in diesen Gebieten kein Punktspektrum haben, so können wir die positiven Konstanten $\delta_{\mu}$ $(\mu=1,2, \ldots, m)$ so klein wählen, dass die gleichzeitigen Bedingungen

$$
\begin{aligned}
& \sum_{\substack{\mu=1 \\
\varrho_{\mu}+\delta_{\mu}>>z-\alpha_{\mu}>\varrho_{\mu}-\delta_{\mu}}}^{m} \varphi_{m}(z, \bar{z}) d\left(E_{x} E_{y} f, f\right) \leqq \frac{\varepsilon}{4}, \\
& \sum_{\substack{\mu=1 \\
\varrho_{\mu}+\delta_{\mu}>\left|z-\alpha_{\mu}\right|>\varrho_{\mu}-\delta_{\mu}}} \int_{m} \int_{m}(z, \bar{z}) d\left(E_{y} E_{x} f, f\right) \leqq \frac{\varepsilon}{4}
\end{aligned}
$$

gelten. 
Wir bezeichnen jetzt mit $\varphi_{K}(z, \bar{z})$ die Funktion, die gleich 1 in $K$ ist und die ausserhalb des Gebietes $K$ verschwindet. Wenn nun die Konstanten $\delta_{\mu}(\mu=1,2, \ldots, m)$ genügend klein gewählt worden sind, erfüllt auch $\varphi_{K}(z, \bar{z})$ die gleichzeitigen Bedingungen

und

$$
\sum_{\mu=1}^{m} \int_{\varrho_{\mu}+\delta_{\mu}>\mid z} \int_{\alpha_{\mu} \mid>\varrho_{\mu}-\delta_{\mu}} \varphi_{K}(z, \bar{z}) d\left(E_{y} E_{x} f, f\right) \leqq \frac{\varepsilon}{4}
$$

$$
\sum_{\mu=1}^{m} \int_{\varrho_{\mu}+\delta_{\mu}>\left|z-\alpha_{\mu}\right|>\varrho_{\mu}-\delta_{\mu}} \int_{K}(z, \bar{z}) d\left(E_{x} E_{y} f, f\right) \leqq \frac{\varepsilon}{4} .
$$

Als Zusammenfassung der obenerwähnten Eigenschaften der Funktion $\varphi_{K}(z, \bar{z})$ können wir nun schliessen, dass

$$
\int_{G} \int\left|\varphi_{m}(z, \bar{z})-\varphi_{K}(z, \bar{z})\right| d\left(E_{x} E_{y} f, f\right)<\varepsilon
$$

und

$$
\int_{G} \int\left|\varphi_{m}(z, \bar{z})-\varphi_{K}(z, \bar{z})\right| d\left(E_{y} E_{x} f, f\right)<\varepsilon
$$

gelten; hierbei bedeutet $G$ die ganze komplexe Ebene. Für $m=\infty$ haben wir also

$$
\lim _{m \rightarrow \infty} \iint_{G} \varphi_{m}(z, \bar{z}) d\left(E_{x} E_{y} f, f\right)=\iint_{G} \varphi_{K}(z, \bar{z}) d\left(E_{x} E_{y} f, f\right)
$$

und

$$
\lim _{m \rightarrow \infty} \iint_{G} \varphi_{m}(z, \bar{z}) d\left(E_{y} E_{x} f, f\right)=\iint_{G} \varphi_{K}(z, \bar{z}) d\left(E_{y} E_{x} f, f\right) .
$$

Unter Berücksichtigung der Gleichung (1.7) erhalten wir

$$
\iint_{G} \varphi_{K}(z, \bar{z}) d\left(E_{x} E_{y} f, f\right)=\iint_{G} \varphi_{K}(z, \bar{z}) d\left(E_{y} E_{x} f, f\right) .
$$

Da $K$ ein beliebiges beschränktes abgeschlossenes einfach zusammenhängendes Gebiet von $G$ ist, bedeutet (1.9), dass die Funktionen $\left(E_{x} E_{y} f, f\right)$ und $\left(E_{y} E_{x} f, f\right)$ einander gleich sowie eindeutige Funktionen von $x$ und $y$ und also von $z=x+i y$ sind, wenn wir $\varrho(z)=\left(E_{x} E_{y} f, f\right)$ setzen.

Aus den Eigenschaften von $E_{x}$ und $E_{y}$ folgt weiterhin, dass $\varrho(z)$ nachstehende Eigenschaften hat: 
(a)

$$
\int_{K} d \varrho(z)=\iint_{K} d\left(E_{x} E_{y} f, f\right) \geqq 0 \text { bei jedem } K \text { von } G,
$$

$$
\begin{aligned}
& \left.\lim \varrho(z)=0, \text { wenn } \operatorname{Re}(z)=\infty \text { und } \operatorname{Im}(z)=-\infty,{ }^{1}\right) \\
& \lim \varrho(z+\zeta)=\varrho(z), \text { wenn } \zeta \rightarrow 0 \text { und wenn } \\
& \operatorname{Re}(\zeta) \geqq 0 \text { und } \operatorname{Im}(\zeta) \geqq 0 .
\end{aligned}
$$

Wir haben in [3] (Nrn. 3.5-3.7; S. 25-26) gezeigt, dass einer solchen Funktion $\varrho(z)$ immer eine eindeutig definierte hypermaximale normale Transformation $N_{1}$ und ihre Adjungierte $\bar{N}_{1}$ entsprechen. Wir haben für jede Funktion $F(z, \bar{z})$ von $z$ und $\bar{z}$, für die das Integral $\int_{G}\left|F^{\prime}(z, \bar{z})\right|^{2} d \varrho(z)$ existiert,

$$
\left(F\left(N_{1}, \bar{N}_{1}\right) f, f\right)=\int_{G} F(z, \bar{z}) d \varrho(z) ;
$$

hierbei bedeutet $F\left(N_{1}, \bar{N}_{1}\right)$ die dem $F(z, \bar{z})$ entsprechende Funktion von $N_{1}$ und $\bar{N}_{1}$ und $f$ das in (1.3) definierte und in (1.i) näher normierte Element in $\mathfrak{X}$.

Wir bezeichnen nun mit $\mathfrak{X}_{1}$ die lineare Menge, die von den Elementen $P\left(N_{1}, \bar{N}_{1}\right) f$ gebildet wird, und mit $\mathfrak{S}_{1}$ den kleinsten abgeschlossenen linearen Unterraum in $\mathfrak{S}$, der $\mathfrak{X}_{1}$ enthält; $P\left(N_{1}, \bar{N}_{1}\right)$ bedeutet hierbei ein beliebiges Polynom von $N_{1}$ und $\bar{N}_{1}$. Aus der Bestimmungsweise der Transformationen $N_{1}$ und $\bar{N}_{1}$ folgt dann, dass $N_{1}$ und $\bar{N}_{1}$ mit $N$ und $\bar{N}$ in $\mathfrak{X}_{1}$ übereinstimmen.

Falls $\mathfrak{S}_{1}$ nicht den ganzen Raum $\mathfrak{F}$ enthält, wählen wir ein normiertes Element $f_{2}$ von $\mathfrak{X}$, das orthogonal gegen $\mathfrak{H}_{1}$ ist, und bezeichnen mit $g_{2}$ das Element, das die Eigenschaft

$$
e^{-\frac{(N \bar{N})^{2}}{2}} f_{2}=g_{2}
$$

hat.

Bezeichnen wir jetzt mit $\mathfrak{X}_{2}$ die von den Elementen $P(N, \bar{N}) g_{2}$ gebildete lineare Mannigfaltigkeit und mit $\mathfrak{h}_{2}$ den kleinsten linearen abgeschlossenen Unterraum von $\mathfrak{S}$, der $\mathfrak{X}_{2}$ enthält: $P(N, \bar{N})$ bedeutet wie oben ein beliebiges Polynom von $N$ und $\bar{N}$. Genau wie oben, können wir nun die hypermaximalen normalen Transformationen $N_{2}$ und $\bar{N}_{2}$ in $\mathfrak{S}_{2}$ bestimmen, die zueinander adjungiert sind und entsprechend mit $N$ und $\bar{N}$ in $\mathfrak{X}_{2}$ übereinstimmen.

$\left.{ }^{1}\right) \operatorname{Re}(\zeta)$ bzw. $\operatorname{Im}(\zeta)$ bedeutet den reellen bzw. imaginären Teil von $\zeta$. 
Falls die Elemente von $\mathfrak{\mathfrak { C }}_{1}$ und $\mathfrak{\mathscr { C }}_{2}$ den ganzen Raum $\mathfrak{H}$ nicht aufspannen, können wir den obigen Prozess wiederholen, so dass wir schliesslich eine Menge von gegeneinander orthogonalen linearen Mannigfaltigkeiten $\mathfrak{X}_{1}, \mathfrak{X}_{2}, \mathfrak{X}_{3}, \ldots$ in $\mathfrak{X}$ und ihren kleinsten linearen abgeschlossenen Fortsetzungen $\mathfrak{\mathfrak { S }}_{1}, \mathfrak{\mathfrak { S }}_{2} \mathfrak{\mathfrak { S }}_{3}, \ldots$ mit der Eigenschaft $\mathfrak{\mathfrak { S }}_{1} \oplus \mathfrak{\mathfrak { g }}_{2} \oplus \mathfrak{\mathfrak { G }}_{3} \oplus \ldots=\mathfrak{H}_{\mathfrak{c}}$ erhalten. In $\mathfrak{S}_{1}, \mathfrak{H}_{2}, \mathfrak{H}_{3}, \ldots$ können wir dann entsprechend die hypermaximalen normalen Transformationen $N_{1}, N_{2}, N_{3}, \ldots$ und $\bar{N}_{1}, \bar{N}_{2}, \bar{N}_{3}, \ldots$ bestimmen, so dass immer $N_{v}$ mit $\bar{N}_{v}\left(l^{\prime}=1,2,3, \ldots\right)$ zueinander adjungiert sind und diese Transformationen mit $N$ und $\vec{N}$ in $\mathfrak{x}_{1}, \mathfrak{x}_{2}$, $\mathfrak{X}_{3}, \ldots$ übereinstimmen.

Bilden wir nun die lineare Transformation $N_{0}$ bzw. $\bar{N}_{0}$, die mit $N_{1}, N_{2}, N_{3}, \ldots$ bzw. $\bar{N}_{1}, \bar{N}_{2}, \bar{N}_{3}, \ldots$ in den entsprechenden linearen Unterräumen $\mathfrak{S}_{1}, \check{\mathfrak{S}}_{2}, \mathfrak{S}_{2}, \ldots$ übereinstimmen. Da die Einschränkungen von $N_{0}$ und $\bar{N}_{0}$ in jedem der gegeneinander orthogonalen Unterräume $\tilde{S}_{1}$. $\mathfrak{\mathfrak { S }}_{2}, \mathfrak{\mathfrak { S }}_{3}, \ldots$ hypermaximal normal sind und diese Unterräume den ganzen Hilbertschen Raum $\mathscr{S}$ aufspannen, so sind $N_{0}$ und $\bar{N}_{0}$ auch hypermaximal normal. $\mathrm{Zu}$ dem gemeinsamen Definitionsbereich von $N_{0}$ und $\bar{N}_{0}$ gehört das ganze $\mathfrak{X}$, und nach dem Obigen stimmen diese Transformationen in $\mathfrak{X}$ mit $N$ und $\bar{N}$ überein.

Dies aber bedeutet, dass die normalen Transformationen $N=P+i Q$ und $\bar{N}=P=i Q$ mit dem gemeinsamen Definitionsbereich $\mathfrak{X}$ zu den hypermaximalen normalen Transformationen $N_{0}$ und $\bar{N}_{0}$ fortsetzbar sind. Da die Transformationen $P$ und $Q$ mit den Definitionsbereichen $D(P)$ und $D(Q)$ schon selbstadjungiert sind, ist die Fortsetzbarkeit nur so möglich, dass die kleinsten linearen abgeschlossenen Fortsetzungen von $N$ und $\bar{N}$ mit $N_{0}$ und $\bar{N}_{0}$ übereinstimmen und also hypermaximal normal sind. Unser Satz ist damit vollständig bewiesen.

\section{§ 2. Die quantenmechanischen Vertauschungsrelationen}

Wie schon in der Einleitung erwähnt, gibt es in der Rellichschen Version des Satzes 1 statt $2^{\circ}$ die Vermutung, dass die Transformation $A=\sum_{j=1}^{n}\left(P_{j}^{2}+Q_{j}^{2}\right) \quad$ zerlegbar ist. Aus dieser Rellichschen Annahme folgt nach Dixuier [1], dass die kleinsten abgeschlossenen linearen Fortsetzungen von $P_{j}^{\prime 2}+Q_{j}^{\prime 2}(j=1,2, \ldots, n)$ selbstadjungiert sind; $P_{j}^{\prime}, Q_{j}^{\prime}$ $(j=1,2, \ldots, n)$ bedeuten die Einschränkungen der Transformationen $P_{j}, Q_{j}(j=1,2, \ldots, n)$ auf $\mathfrak{x}$. Daraus folgt weiterhin, dass die kleinsten abgeschlossenen linearen Fortsetzungen von $P_{j}^{\prime}$ und $Q_{j}^{\prime}(j=1,2, \ldots, n)$ selbstadjungiert sind (vgl. Dixmier [1], S. 267). 
Wir werden jetzt zeigen, dass die Dixmiersche Annahme $2^{\circ}$ des Satzes 1 durch folgende ersetzt werden kann, ohne die Behauptungen dieses Satzes einzuschränken:

$2^{\circ \circ}$. Die kleinsten abgeschlossenen linearen Fortsetzungen der auf $\mathfrak{X}$ liegenden Einschränkungen von $P_{j}^{2}+Q_{j}^{2}(j=1,2, \ldots, n)$ sind selbstadjungiert.

Beweis. Nach der Annahme des Satzes 1 gelten die Bedingungen

$$
\begin{aligned}
& \left|\left(P_{j}+i P_{k}\right) f\right|^{2}=\left|\left(P_{j}-i P_{k}\right) f\right|^{2} \\
& \left|\left(Q_{j}+i Q_{k}\right) f\right|^{2}=\left|\left(Q_{j}-i Q_{k}\right) f\right|^{2} \quad j, k=1,2, \ldots, n ; j \neq \mathrm{k}, \\
& \left|\left(P_{j}+i Q_{k}\right) f\right|^{2}=\left|\left(P_{j}-i Q_{k}\right) f\right|^{2}
\end{aligned}
$$

bei jedem $f$ von $\mathfrak{X}$. Da die kleinsten abgeschlossenen linearen Fortsetzungen von $P^{\prime}$ und $Q^{\prime}$ selbstadjungiert sind, folgt aus den obigen Gleichungen, dass die Transformationen $P_{j}^{\prime}+i P_{k}^{\prime}, Q_{j}^{\prime}+i Q_{k}^{\prime}, P_{j}^{\prime}+i Q_{k}^{\prime}$ $(j, k=1,2, \ldots, n ; j \neq k)$ mit den in $\mathfrak{S}$ dichten Definitionsbereich $\mathfrak{X}$ normal und ihre kleinsten abgeschlossenen linearen Fortsetzungen nach Satz 2 sogar hypermaximal normal sind.

Bezeichnen wir jetzt mit $T$ die kleinste abgeschlossene lineare Fortsetzung von $P_{1}^{\prime}+i P_{2}^{\prime}$. Wir haben also

$$
T=\left(P_{1}^{\prime}+i P_{2}^{\prime}\right)^{* *}, \quad T^{*}=\left(P_{1}^{\prime}-i P_{2}^{\prime}\right)^{* *} .
$$

Da $T$ hypermaximal normal ist, ist die Transformation $T * T$ selbstadjungiert. Sei nun $g$ ein beliebiges Element des Definitionsbereiches $D\left(T^{*} T\right)$ von $T^{*} T$. Aus der Bestimmungsweise von $T^{*} T$ folgt, dass wir aus $\mathfrak{X}$ eine solche Reihe $g_{1}, g_{2}, \ldots, g_{m} \ldots$ wählen können, bei der gleichzeitig

$$
\lim _{m \infty \rightarrow} g_{m}=g
$$

und

$$
\lim _{m \rightarrow \infty}\left(P_{1}^{\prime}-i P_{2}^{\prime}\right)\left(P_{1}^{\prime}+i P_{2}^{\prime}\right) g_{m}=\lim _{m \leftarrow \infty}\left(P_{1}^{\prime 2}+P_{2}^{\prime 2}\right) g_{m}=T^{*} T g
$$

gelten.

Aus der Existenz des Grenzwertes $\lim _{m \rightarrow \infty}\left(P_{1}^{\prime 2}+P_{2}^{\prime 2}\right) g_{m}$ folgt dann, dass das Element $\lim _{m \rightarrow \infty} g_{m}=g$ zum Definitionsbereich der kleinsten abgeschlossenen linearen Fortsetzung von $P_{1}^{\prime 2}+P_{2}^{\prime 2}$ gehört. Wir haben also

$$
\left(P_{1}^{\prime 2}+P_{2}^{\prime 2} \supseteq T^{*} T\right. \text {. }
$$


Da $P_{1}^{\prime 2}+P_{2}^{\prime 2}$ symmetrisch ist, bedeutet die obige Bedingung, dass ihre kleinste abgeschlossene lineare Fortsetzung $\left(P_{1}^{\prime 2}+P_{2}^{\prime 2}\right)$ mit $T^{*} T$ übereinstimmt und also selbstadjungiert ist. Analog lässt sich beweisen, dass die kleinsten abgeschlossenen linearen Fortsetzungen der Transformationen $P_{j}^{\prime 2}+P_{k}^{\prime 2}, Q_{j}^{\prime 2}+Q_{k}^{\prime 2}, P_{j}^{\prime 2}+Q_{k}^{\prime 2}(j, k=1,2, \ldots, n ; j \neq k)$ selbstadjungiert sind.

Wir können also, ohne die Behauptung des Satzes 1 einzuschränken, die Annahme $2^{\circ}$ durch $2^{\circ \circ}$ ersetzen, und damit ist alles bewiesen. 


\section{Literatur}

[1] Dixmen, J.: Sur la relation $i(P Q-Q P)=1$. Compositio Math. 13 (1958), S. $263-269$.

[2] Fotas, C.-GehÉr, L.-Sz.-NAGY, B.: On the permutability condition of quantum mechanics. - Acta Sci. Math. Szeged XXI (1960), S. 78-89.

[3] Kulpi, Y.: Über das komplexe Momentenproblem. - Ann. Acad. Sci. Fenn. A I, 236 (1957).

[4] Reurrch, F.: Der Eindeutigkeitssatz für die Lösungen der quantenmechanischen Vertauschungsrelationen. - Göttinger Nachr. (1946), S. 107-116.

[5] Stone, M. H.: Derelopments in Hermite polynomials. - Ann. of Math. 29 (1928). S. $1-13$.

[6] -»- Linear transformations in Hilbert space. - New York. 1932. 